\title{
Transformatorische Fluchtverläufe: Migration aus lokalisierten Milieus und Etablierung in einem neuen Kontext am Beispiel irakischer Geflüchteter in Jordanien
}

\author{
Johannes Becker, Hendrik Hinrichsen
}

\section{Einleitung}

In unserem Beitrag stellen wir den transformatorischen Charakter von Fluchtprozessen ins Zentrum. Wir zeigen auf, dass Flucht ${ }^{1}$ mit manchmal abrupten, oft jedoch schleichenden kollektiv- und lebensgeschichtlichen Prozessen einhergeht, in denen sich Erfahrungen, Deutungsmuster, Kapitalausstattungen und Netzwerke der Flüchtenden erheblich verändern können. Während für Fluchtprozesse innerhalb des arabischsprachigen Nahen Ostens oft auf die Vorteile regionaler Ähnlichkeiten sowie auf die daher leichte und erfolgreiche Etablierung von Geflüchteten an „neuen“ Orten hingewiesen wird, werden wir im Folgenden einen Typus von Geflüchteten umreißen, der zutiefst durch den transformatorischen Charakter von Fluchtprozessen bestimmt ist und durch einschneidende Erfahrungen von Fremdheit in neuen geographischen Kontexten. Dabei steht der transformatorische Charakter der Fluchtdy-

1 Wir verstehen Flucht im Anschluss an Worm (2019) als Migrationsprozess, der durch Gewalt- und Konfliktdynamiken strukturiert ist. Flüchtende versuchen, sich Gewaltdynamiken zu entziehen, bleiben häufig jedoch über die ,unmittelbare“ Flucht hinaus mit diesen verwoben. 
namik mit einer Verortung in einem stark lokalisierten Milieu vor der Migration im Zusammenhang - dies ist der zentrale, von uns im Folgenden entfaltete Gedankengang.

Den Typus mit seinen Aspekten transformatorischen Wandels und dessen Folgen für Etablierungen diskutieren wir auf Basis unserer Erhebungen im Kontext des DFG-Forschungsprojektes „Dynamische Figurationen von Flüchtlingen, Migranten und Altansässigen in Jordanien seit 1946: Zwischen erfolgreichem und konfliktreichem Zusammenleben?" ${ }^{\text {" }}$ Unsere Analysen zeigen ein Zusammenwirken verschiedener lebensgeschichtlicher und kollektivgeschichtlicher Prozesse, das im Verlauf der Flucht zu sozialem Abstieg, Kapitalverlust, langwierigen körperlichen und seelischen Verletzungen und insbesondere zur unbeabsichtigten Immobilisierung in Amman beiträgt. Auf Basis dieser Analysen kritisieren wir in diesem Beitrag einerseits eine grobmaschige Annahme eines alle Milieus oder Schichten umgreifenden arabischen Regionalismus. Andererseits gehen wir auf einen Aspekt ein, der in der jüngeren Literatur zu Fluchtbewegungen (vgl. Etzold 2019) besonders hervorgehoben wird: Die Bedeutung von „refugee journeys“ (BenEzer/Zetter 2015) bzw. generell von Fluchtprozessen im lebens- und soziohistorischen Kontext (Worm 2019). „Refugee journeys" durchdringen noch das Leben und die Etablierung in einem neuen geographischen Kontext und sind mit Fragen der (Im-)Mobilität von Geflüchteten verknüpft. Auf diese beiden Aspekte - die Bedeutung von Flucht und die Regionalismuskritik - gehen wir im Folgenden kurz ein.

Die vorherige Flucht gestaltet das Leben in einem neuen Ort - neben anderem - entscheidend mit. Natürlich sind die rechtlichen Rahmenbedingungen im neuen Kontext oder die Figurationen mit anderen Gruppierungen dort relevant. Aber die Genese der Fluchtsituation (in ihrer Differenz zur vorherigen Lebenssituation), das Erleben der Flucht, deren Verlauf, körperliche, psychische und soziale Folgen wirken sich unmittelbar auf den Etablierungsprozess in einem neuen Ort aus und tragen zu differierenden Chancen dort bei. Auch der Fluchtverlauf kann also unterschiedliche Orientierungen von Flüchtlingen nach der Ankunft in einem neuen Ort hervorrufen. Wir sprechen in diesem Zusammenhang vom transformatorischen Charakter von Fluchtprozessen. Das heißt, die unterschiedlichen Orientierungen sind nicht alleinig durch die Analyse von Schichtzugehörigkeit oder angesammeltem kulturellem und sozialem Kapital im Herkunftskontext zu verstehen, die zwar für Fluchtprozesse relevant sind, aber gleichzeitig durch die Flucht erschüttert werden können. Vielmehr legen wir einen besonderen Fokus auf „refugee journeys":

The process and meaning of the journey will almost always have a transformative effect on lives during the journey and will mediate the wayfarers' experience of arrival at the new country of residence, whether exile or return. ... In this way it should be possible to develop and elaborate a conceptualization of the exilic journey as an agency of change and a transformative event in the lives of refugees. A disregard for refugee journeys and

${ }^{2}$ Kennziffer: RO 827/20-1; das von April 2017 bis Februar 2022 laufende Projekt wird von Prof. Gabriele Rosenthal (Göttingen) geleitet. 
the complex processes involved may result in limited or, more likely, erroneous interpretation of the refugees' state in their new country. (BenEzer/Zetter 2015: $302 \mathrm{f}$.)

Gleichzeitig gehen wir davon aus, dass diese so notwendige Analyse von „refugee journeys“ nicht ohne die Einbindung dieser „Reisen“ in die lebens- und familiengeschichtlichen Perspektiven in ihrer Tiefe geleistet werden können. Wir bedienen uns daher insbesondere einer biographietheoretischen, prozessorientierten Perspektive auf Flucht- und Etablierungsprozesse (Rosenthal/Worm/Bahl 2016; Worm 2019). Mit einer solchen Perspektive wird einerseits erkennbar, wie Fluchtprozesse durch die Lebensbedingungen vor der Flucht strukturiert sind und in welcher Weise sie mit den sich wandelnden kollektivgeschichtlichen Prozessen während der Flucht verwoben sind. Anderseits zeigt sich in dieser Perspektive auch, dass Fluchtprozesse häufig familiale Projekte sind. Sie sind von den Verhältnissen in der erweiterten Familie und in der Kernfamilie - natürlich wiederum rückgebunden an kollektivgeschichtliche Prozesse - beeinflusst. In der im Folgenden vorgestellten Analyse nehmen wir daher die Fallebene einer Kernfamilie ein. Wir haben in der hier vorgestellten irakischen Familie fünf ausführliche biographisch-narrative Interviews mit Familienmitgliedern in zwei Generationen geführt und können somit auf besonders dichtes empirisches Material zurückgreifen, um die Flucht als familiales Projekt zu diskutieren und die damit verbundenen familialen Dynamiken vorzustellen.

Wir stellen diese Analysen in einem Süd-Süd-Kontext an, am Beispiel der Flüchtlingsbewegung vom Irak nach Jordanien. Damit ist mit Fluchtmigration innerhalb des arabischsprachigen Nahen Ostens einerseits ein regionaler Kontext angesprochen, der zum Beispiel im panarabischen Nationalismus als zusammengehörig wahrgenommen wurde. Dessen Bewohner*innen hätten dieser Auffassung nach mehrheitlich die gleiche „ethnische“ Zugehörigkeit, teilten trotz unterschiedlicher Dialekte eine Sprache, Stämme umspannten weite Teile der Region, es gebe eine lange Geschichte des regionalen Austausches und der Migration. „Translokalität“ meint hier also Transfer und Austausch über verschiedene mögliche Grenzen vor dem Nationalstaat und jenseits des Nationalstaates hinweg (Freitag/von Oppen 2010). Die Ländergrenzen werden in dieser Sichtweise als künstliche Setzung der ehemaligen Kolonialmächte interpretiert. Darauf aufbauend wird angenommen, dass Flüchtenden - wenn sie nicht in Flüchtlingslagern leben - dieser Umstand beim Ankommen im neuen lokalen Kontext entgegenkommt: Einfache Verständigung, Anschließen an bestehende Netzwerke, geringes Erleben von Fremdheit in angrenzenden Ländern. Translokale Mobilität und Verortung konterkarieren in dieser Sichtweise die Bedeutung der Nationalstaaten bzw. Nationalgrenzen (Chatelard 2010a; El-Abed 2014; Shami 1996). In diesem Sinne fasst Giulia El Dardiry (2017: 704) die Bedeutung dieses „,regionalist paradigm“ für Flüchtlingsbewegungen innerhalb des arabischsprachigen Nahen Ostens zusammen:

Recent scholarship on the Iraqi and Syrian displacements has highlighted how places of exile are conceptualized and experienced as familiar; how displacement has overlain long-established crossborder labor migration routes; how Arabism and religious identi- 
ties ... have shaped migration trajectories; and how regional understandings of hospitality and asylum have enabled refugees to self-settle in new places. This regionalist paradigm has been particularly important for research on Jordan, a country with intense movements into and across its territory ... This focus on connection over fragmentation has troubled ahistorical and state-centric notions of belonging.

Die Analysen im Rahmen unseres Forschungsprojektes bestätigen diese ,,regionalistischen" Annahmen durchaus. Sie zeigen sich in einem tendenziell dominanten Typus von Geflüchteten. So konnten wir in unserem Sample einerseits diese relativ erfolgreiche Etablierung im Rahmen eines Typus des wenig transformatorischen Fluchtverlaufs und der erfolgreichen Etablierung in Amman rekonstruieren, der insbesondere auf (historisch zurückreichender) translokaler Einbindung von Familien und auf Netzwerkbindungen beruht (Becker im Druck; Becker/Hinrichsen/Worm im Druck). Eine generalisierte Annahme einer relativ erfolgreichen Etablierung aufgrund der Ähnlichkeit der „Herkunfts-“ und „Ankunfts-“"kontexte und aufgrund translokaler Einbindung ist jedoch deutlich zu kurz gegriffen. ${ }^{3}$

Die generalisierte Annahme von translokalen Verbindungen und der Wahrnehmung von Ähnlichkeiten verneint nämlich die Möglichkeit von regional stärker gebundenen (Familien-)Biographien oder Milieus. ${ }^{4}$ Geflüchtete bzw. ihre Familien verfügen dann über wenig ,,translokales Kapital“, ihre Handlungsmuster sind wenig mit Praktiken der translokalen Mobilität verknüpft, sie haben wenige regionenübergreifende Netzwerke ausgebildet - sie sind kaum über die Herkunftsprovinzen hinaus, also in Bezug auf Mobilität und Netzwerke weder transnational noch national integriert. Unsere dahingehenden Analysen in diesem Beitrag kritisieren somit die soeben vorgestellte Regionalismusthese, stimmen aber in einem Aspekt mit ihr überein; nämlich darin, die Bedeutung des Nationalstaates einzuhegen - im Falle der Regionalismusthese durch die Betonung der Integration auf dem Level des arabischsprachigen Nahen Ostens, in unserem Beitrag durch die von uns analysierte Beschränkung der Integration auf ein bestimmtes, regional oder lokal verankertes Milieu. In unseren Fallanalysen wird deutlich, dass für eher regional verortete Familien selbst der Umzug in eine andere Provinz bereits eine Erfahrung starker struktureller Fremdheit bedeuten kann, Erfahrungen ,außerhalb einer bestimmten Ordnung“ (Waldenfels 1997: 36). Damit verknüpft ist die Frage, welche lebensweltlichen Folgen die Flucht hat, ob zum Beispiel durch eine lange Migrationsroute durch fremde lokale Kontexte translokales Kapital aufgebaut werden kann, die Milieuverankerung oder soziale Netzwerke aber abhandenkommen können. Hieraus speisen sich

3 Die translokale Einbettung ist für Flüchtlinge wichtig für die Frage, in welche Region sie fliehen können, ob und in welchem Ausmaß sie sich in den Aufnahmeregionen etablieren können und ob sie eine Weitermigration innerhalb des Nahen Ostens oder in andere Weltregionen anstreben und umsetzen können (Becker/Hinrichsen/Worm im Druck).

${ }^{4}$ Hier verstanden als soziale Makro-Milieus. Für eine Diskussion des Milieubegriffs und dessen Anwendung auf gesellschaftliche Kontexte im Globalen Süden vgl. Neubert/Stoll 2015; Rehbein/Stoll 2017. 
Unterschiede, die die Flucht in translokal vernetzten Fällen relativ „problemlos“ machen können, während sie für andere lange, erschöpfend und transformatorisch ist.

Aber auch die Nationalgrenzen im Nahen Osten haben in den vergangenen Jahrzehnten vermehrt an administrativer Relevanz für Regulierung und Einschränkung von Mobilität und an Bedeutung als Grundlage von Selbst- und Fremddefinitionen gewonnen und müssen trotz deren „kurzer" Geschichte als relevanter Faktor anerkannt werden.

Wir diskutieren die Bedeutung von transformatorischen Fluchterfahrungen für das Erleben späterer „Stationen“ der Flucht beispielhaft am Fall einer nach Amman geflohenen Familie aus dem Irak. Der Typus, den die Familie verkörpert, steht für einen Teil unseres Samples in Amman. Die Aufgabe des sozialen Lebens, der komplexe, oft traumatisierende Fluchtprozess, der soziale Abstieg, die alternativlose Immobilität in illegalisierten Verhältnissen haben wir bei jenem Teil der irakischen und syrischen Flüchtlinge, die noch unter prekären und besonders ungesicherten Bedingungen in Amman wohnen, als einigende Elemente ausgemacht. Am Beispiel der Familie Abu Uthmans können sie besonders gut aufgezeigt werden. Wir haben fünf Familienmitglieder interviewt - die beiden Eltern Abu Uthman und Um Uthman (beide geb. in der ersten Hälfte der 1970er Jahre) sowie die älteren Kinder Uthman (geb. 1995), Samar (geb. 1996) und Aya (geb. 2000). Die Interviews dauerten insgesamt 15 Stunden. ${ }^{5}$ Wir verfolgen zunächst den komplexen Fluchtprozess der Familie nach der US-amerikanischen Besatzung des Iraks 2003. Nach forciertem Systemwechsel und Entbaathisierung (dem Ausschluss derjenigen aus dem öffentlichen Dienst, die Verbindungen zur vormals herrschenden Baath-Partei hatten) konfessionalisierten sich die Gewaltprozesse, die insbesondere von Milizen getragen wurden. Die Familienmitglieder entzogen sich diesen Prozessen mit mehrfachen Ortswechseln bzw. Binnenmigrationen im Irak. Sie machten in jener Phase massive Gewalterfahrungen, mit denen traumatische biographische Episoden begannen. Im Zuge der Flucht zerfielen die Bildungskarrieren der Kinder; Ehefrau und selbst jüngere Kinder trugen zunehmend zur Grundversorgung der Familie bei. Die Kernfamilie trat als Einheit hervor und war zunehmend auf sich gestellt. Die Verbindungen zur erweiterten Familie in der Herkunftsregion begannen sich bereits im Zuge der Binnenmigration abzuschwächen und später zunehmend zu lösen, auch weiteres soziales Kapital ging verloren. Die Familie verfügte über immer weniger Besitz, körperliche und seelische Folgen der langen Flucht setzten sich fest. Gleichzeitig eröffneten

\footnotetext{
${ }^{5}$ Das erste Interview mit dem Vater Abu Uthman führte Johannes Becker mit Marwan Nabil Muhammad über eine Dauer von zweieinviertel Stunden. Darauf folgten zwei Interviewtreffen mit der Mutter Um Uthman, die Johannes Becker gemeinsam mit Samira Qatuni führte (insgesamt viereinhalb Stunden). Hendrik Hinrichsen interviewte gemeinsam mit Samira Qatuni die Töchter Aya (drei Stunden) und Samar (zweieinhalb Stunden). Johannes Becker führte wiederum mit Marwan Nabil Muhammad das Interview mit dem Sohn Uthman (zweidreiviertel Stunden). Alle Interviews fanden im Zeitraum von Ende Oktober bis Mitte Dezember 2018 in Amman statt und wurden teilweise direkt auf Arabisch und teilweise mit Übersetzung geführt. Außerdem führten wir fünf Interviews in der palästinensischjordanischen Familie, in die zwei von Abu und Um Uthmans Kindern in Amman hineingeheiratet haben. Namen und weitere Details wurden von uns zum Zwecke der Anonymisierung verändert.
} 
sich den Kindern und der Ehefrau im Fluchtverlauf im Vergleich zum Leben vor der Flucht Chancen auf Autonomiegewinne. Dies alles sind keine abrupten Veränderungen, sondern relativ langfristige, schleichende Prozesse, die sich im Zuge der Flucht in der Familie verankerten. Sie strukturierten auch die spätere Phase in Amman, wo die Mitglieder der Kernfamilie sukzessive eintrafen. Amman war zunächst als Zwischenetappe vorgesehen, wurde aber zum verstetigten Lebensmittelpunkt der Familie. Zur prekären Situation in Amman trugen einerseits die benachteiligenden Aufenthaltsregelungen für Iraker*innen in Jordanien bei, andererseits speiste sich diese jedoch aus den genannten schleichenden transformatorischen Prozessen. Aufenthaltsregelungen und transformatorische Prozesse führten zusammengenommen zum Absinken der Familie aus dem bäuerlich geprägten Milieu in eine Art urbanes Subproletariat und somit zu einer Milieuangleichung an die jordanische Unterschicht. So wurde die weitgehende Immobilisierung der Familie hergestellt, deren Hoffnung auf Weitermigration sich nicht erfüllte, die jedoch auch nicht in den Irak zurückkehren kann. In der Folge richtete sich die Familie langsam in der prekären Situation in Amman ein, was auch mit Heiraten in die „abgehängte“ Ammaner Unterschicht einherging. Diese ineinandergreifenden Prozesse detaillieren wir in der folgenden Falldarstellung, in der wir die kollektivgeschichtlichen und familiengeschichtlichen Kontexte sowie die komplexen Routen und die Stationen der Flucht nachvollziehbar machen.

\section{Milieugebundenes Leben vor 2003}

Um die drastische Auflösung sozialer Strukturen im familialen Zusammenhang der Familie Abu und Um Uthmans nachzuvollziehen, gehen wir kursorisch auf die familiengeschichtliche und soziohistorische Situation vor der US-Invasion von 2003 ein. Viele Mitglieder der Herkunftsfamilien von Abu und Um Uthman waren seit mehreren Generationen als Bauern im Gouvernement Diyala ansässig. Diese sich nordöstlich von Bagdad bis an die iranische Grenze erstreckende Provinz ist von landwirtschaftlicher Nutzung geprägt. Die Familien von Abu und Um Uthman wohnten im bereits bäuerlich geprägten Randbezirk der Gouvernementshauptstadt Baquba. Die Einwohner*innen des Gouvernements waren mehrheitlich sunnitischarabisch, die Bevölkerung setzte sich zudem aus ethnischen bzw. religiösen Minderheiten von Kurd*innen, Turkmen*innen und Schiit*innen zusammen, die auf unterschiedliche Weisen während der Regierungszeit Saddam Husseins benachteiligt wurden. Die Mutter Abu Uthmans ist Schiitin genauso wie Ehepartner*innen von Abu und Um Uthmans zahlreichen Geschwistern. Die Familie wohnte in der Nähe des Flusses Sirwan in einer sunnitisch geprägten Nachbarschaft, auf der anderen Flussseite befand sich eine schiitisch geprägte Nachbarschaft. Die von uns interviewten Familienmitglieder, die sich als Sunnit*innen verstehen, betonen die frühere handlungspraktische Irrelevanz der konfessionellen Zugehörigkeit. Tatsächlich gab es im modernen Irak alltäglichen Austausch zwischen Sunnit*innen und Schiit*innen, 
häufig werden als Beispiel die vielen interkonfessionellen Heiraten genannt. In der Baath-Periode wurde die Relevanz konfessioneller Zugehörigkeit hinter die nationale irakische „Identität“ zurückgedrängt und im Alltag wenig offen thematisiert. Differenzen wurden nicht diskutiert, sondern nach außen negiert, gleichzeitig aber innerhalb der eigenen Gruppierung latent oder in Andeutungen weitergegeben (Haddad 2013).

Abu Uthman und Um Uthman wurden in der ersten Hälfte der 1970er Jahre in recht stabile familiale und dörfliche bzw. regionale Verhältnisse geboren. Thre jeweiligen Herkunftsfamilien gehören derselben erweiterten Familie in der Umgebung Baqubas an. Ihre Väter arbeiteten als Bauern und besaßen Land. In den erweiterten Familien - sowohl Um als auch Abu Uthman hatten sieben Geschwister - finden sich neben Bauern auch Soldaten und Familienmitglieder, die in umliegenden gröBeren Städten wohnen. Es ist aber nichts über im Ausland lebende Familienmitglieder bekannt - die Reichweite der Familie scheint auf die zentralirakische Region um Bagdad beschränkt. Die Mischung aus landwirtschaftlicher Arbeit, Einbindung in die mit sozialen Sicherheiten verbundene Armee sowie selbständige und abhängige, oft einfache Tätigkeiten im Dienstleistungssektor, auch in den Städten der Umgebung, ergeben das Bild eines Einkommensmixes, wie etwa von Georg Elwert, HansDieter Evers und Werner Wilkens (1983) als „kombinierte Produktionsformen“ diskutiert. Diese „Kombination“ dient dazu, Risiken z.B. durch schlechte Ernten, Naturkatastrophen oder politische Entwicklungen zu begegnen. Auch Abu Uthman verlängerte seinen obligatorischen Wehrdienst, während dem er den Zweiten Golfkrieg miterlebte, und betrieb in einer Kaserne ab 1992 ein Lebensmittellädchen, das er 1995 verkaufte, um in Diyala zu heiraten und zur landwirtschaftlichen Arbeit zurückzukehren. Seine Frau hatte zuvor bei ihren Eltern gelebt. Zuvor waren bereits zwei Brüder Abu Uthmans mit zwei Schwestern Um Uthmans verheiratet worden, was die familienorientierte und lokal geprägte Zukunftsorientierung der Familie aufzeigt. Zwischen 1995 und 2001 bekamen Abu Uthman und Um Uthman vier Kinder, zwei Jungen und zwei Mädchen.

\section{Der Bruch mit der US-Invasion 2003 und die Entwicklung eines komplexen Fluchtprozesses}

Alle unsere irakischen Interviewpartner*innen berichten vom Zusammenbruch vorheriger Strukturen und Gewissheiten im Zuge der US-Invasion 2003 - sei es in Basra, Mosul, Bagdad oder Baquba. Zwar war der Irak in den Jahrzehnten zuvor andauernd in kriegerische Konflikte verwickelt, die Bedeutung von 2003 als Interpretationspunkt liegt aber im Systemwechsel und im Machtvakuum durch die Entbaathisierung, die mit der Auflösung der staatlichen und Verwaltungsstrukturen einhergingen. Die sich anschließenden Phasen der Unsicherheit und der Gewalt brachen in den folgenden Jahren in der Form von Überfällen, Entführungen, Morden 
oder Anschlägen meist unvorhersehbar und abrupt in den Alltag der Interviewpartner*innen ein. Später konfessionalisierten sich die Konflikte zunehmend.

In unseren Interviews mit den Familienmitgliedern Abu und Um Uthmans, aber generell in irakischen Familien, stellte die Rekonstruktion der Gewalteskalation und des Fluchtprozesses eine schwierige Aufgabe dar. Einerseits deutet das darauf hin, wie einschneidend der Einbruch der Gewalt in den Alltag vieler Familien nach 2003 war, und auf das schiere Ausmaß der Gewaltakte in manchen regionalen Kontexten. Andererseits ist damit verbunden, dass die Fluchtverläufe aus dem Irak oft nicht linear waren, sondern komplex, über mehrere Stationen führten, mit Binnen- oder Pendelmigration verknüpft waren und mit längeren Perioden der Unsicherheit, wohin die Fluchtroute führen würde (vgl. Chatelard 2010b).

In allen fünf Interviews mit Mitgliedern der Familie Abu und Um Uthmans nimmt die Zeit zwischen 2003 und der Migration nach Amman zwischen 2010 und 2014 einen zentralen Teil der Selbstpräsentation ein. Die Familienmitglieder berichten auch auf Nachfrage detailliert zu dieser Periode. Doch die Abfolge der Migrationen und der Ereignisse, die letztlich zum Umzug aller Familienmitglieder nach Amman führten und die sich über circa elf Jahre erstreckten, erscheint in abweichenden Varianten - mit unterschiedlichen Abfolgen, Zeit- und Ortsangaben. ${ }^{6}$ Wir haben die Darstellungen der Zeit nach 2003 in ihrem Verhältnis mit politischen Ereignissen oder rechtlichen Bestimmungen abgewogen und stellen in groben Zügen die wahrscheinlichste Abfolge der Ereignisse kurz dar.

Ein halbes Jahr nach Beginn der amerikanischen Besatzung des Iraks im Jahre 2003 zog die Kernfamilie aus ihrer Nachbarschaft in die angrenzende Region Bagdad. Abu Uthman führt als Grund dafür die schlechte Sicherheitslage an. Im ethnoreligiös gemischten Gouvernement Diyala - und insbesondere in der näheren Umgebung der Familie, in der Sunniten und Schiiten besonders nah beieinander wohnten - begannen bereits kurz nach der Invasion die (wohl auch von außen hineingetragenen) Kämpfe um Einflusssphären, die sich durch Gewalt und Einschüchterungen auf das Alltagsleben der Bewohner*innen auswirkten. Die zunehmend polarisierten Konfessionszugehörigkeiten wurden biographisch relevant. Morde und Anschläge nahmen in den Jahren nach 2003 stetig zu, aber auch Drohungen (z.B. an Hauswänden), Einschüchterungen oder Scheidungen von gemischtkonfessionellen Ehen.

Ungefähr ein weiteres halbes Jahr später, als ihnen die Lage auch in Bagdad unsicherer erschien, zog die Familie in die ca. 200 Kilometer entfernte sunnitisch geprägte Provinz Salah ad-Din. Die Familienmitglieder bewirtschafteten dort Land selbst, aber arbeiteten auch für ansässige Bauern. Alle beschreiben die negativen

\footnotetext{
${ }^{6}$ Dies deutet auf mehreres hin: Zum einen nimmt diese Periode einen zentralen Ort in den Erinnerungen der Familienangehörigen ein, aber wahrscheinlich ohne dass sie häufig darüber sprechen; es scheint auch kein aktiv gepflegtes Familiengedächtnis zu geben. Wahrscheinlich ist damit auch eine (sequenzielle) Traumatisierung von Familienmitgliedern verknüpft, wie die Analyse verdeutlichen wird. Es scheint außerdem Geheimnisse zu geben, die Teilen der Familie verborgen sind oder die außerhalb der Familie nicht thematisiert werden.
} 
Erlebnisse in dieser Periode. Die Erinnerungen sind geprägt von der ablehnenden Haltung und der fehlenden Solidarität der dortigen Behörden und Einwohner*innen, die sie als klare Diskriminierung erlebten, von ihrem mangelnden Fachwissen, wie man den anders beschaffenen Boden bewirtschaftet und dem Scheitern daran, von der dem ungewohnten Klima geschuldeten Erkrankung und längeren Arbeitsunfähigkeit Abu Uthmans. Die älteren Kinder (der älteste Sohn Uthman war ca. 9 Jahre alt) besuchten die Schule immer unregelmäßiger, weil sie verstärkt bei der Arbeit helfen mussten, zum Beispiel die Erzeugnisse verkaufen. Der Einbezug der jungen Kinder in die Erwerbsarbeit und der dadurch unterbrochene Schulbesuch charakterisiert auch die weiteren Fluchtstationen innerhalb des Iraks und insbesondere die spätere Station Amman.

Obwohl innerhalb des Iraks und in einer sunnitischen Umgebung, machte die Familie drastische Erfahrungen von Fremdheit, wie Abu Uthman mit deutlichen Worten beschreibt:

Das Leben dort ist schwer, schwer, sehr schwer, der Fremde bleibt Fremder unter ihnen. Du kannst nicht sagen, ich habe hier Freunde oder habe dies und jenes. Nein, sie behandeln dich wie einen Fremden, du bist aus anderen Provinzen und nicht aus deren Heimat.

Dies verstärkte den Rückbezug der Kernfamilie auf sich selbst, da sie als separate Einheit zum ersten Mal so deutlich hervortrat. Auch dieser Fokus auf die Kernfamilie wird in Amman zentral sein. Damit verknüpft gibt es Hinweise darauf, dass bereits die Migration der Kernfamilie nach Salah ad-Din von Mitgliedern der erweiterten Familie in Diyala kritisiert und abgewertet wurde. Für sie sei das Verlassen des „eigenen Grund und Bodens“ schändlich und inakzeptabel gewesen, wie die Tochter Aya über die Haltung der Verwandten im Interview berichtet:

[Die Verwandten] sind nicht ausgewandert, weil sie sagen: „Wie sollen wir im Ausland leben? Wir können nicht [woanders] leben, wie sollen wir unsere Häuser und unsere Ländereien hier verlassen?" Aus diesem Grund sind sie nicht ausgewandert. Mein Vater ... nahm Geld von meinem Großvater und wanderte aus ... Mein Vater hat doch eine sehr schwierige Situation [auf der Flucht] erlebt. Dann sagte er [ihnen, also] sie haben viel Geld seine Brüder, er sagte ihnen: „Schick mir doch [Geld] ..., meine Kinder haben nichts zu essen, damit sie was essen und so." Keiner schickte ihm etwas und keiner half ihm und keiner hörte auf ihn, bei Gott.

Die Periode in Salah ad-Din verdeutlicht somit auch die vorherige, regionalräumlich begrenzte Orientierung der Familie und die wenigen Verbindungen sogar innerhalb des Iraks.

Ungefähr im Jahr 2006, nach zwei bis drei Jahren in Salah ad-Din, kehrte die Familie, nachdem es hieß, es sei dort sicherer geworden, zunächst nach Bagdad und anschließend nach Diyala zurück. Sie zog in eine Wohnung der Familie in einem inzwischen sunnitisch geprägten, vormals diversen Viertel in der Stadt Baquba, aus dem Schiiten vertrieben worden waren (The Oklahoman 2007). Doch nach 2006 wurde die Region durch die gewaltvolle Auseinandersetzung zwischen al-Qaida und 
schiitischen Milizen und durch andauernde Kampagnen gegen die Zivilbevölkerung erschüttert. Mitglieder von al-Qaida nutzten zum Beispiel Hütten in den Feldern von Abu Uthmans Familie als Rückzugsort. Es kam zu militärischen Offensiven der USArmee und der irakischen Armee, die die Milizen bekämpften (Diyala Campaign). In dieser Periode, ca. vier Monate nach der Rückkehr - vielleicht während einer der US-Razzien -, wurde Abu Uthman von US-Truppen gefangengenommen und verhört, später an die irakischen Sicherheitsbehörden übergeben und als Krimineller inhaftiert. Er wurde sowohl von schiitischen irakischen als auch von amerikanischen Einheiten schwer gefoltert (Nahrungs- und Flüssigkeitsentzug, körperliche Misshandlung). Nach ca. drei Monaten kaufte sein Vater ihn frei.

$\mathrm{Ab}$ diesem Zeitpunkt divergieren die Darstellungen der Familienmitglieder in den Interviews noch stärker. Abu Uthmans Angaben zufolge habe er nach seiner Haft mit seiner Familie in Bagdad gewohnt und als Fahrer gearbeitet. Bei einem Behördengang sei deutlich geworden, dass er als gesuchte Person gegolten habe. Kurz danach sei er, noch im Jahr 2006, auf direktem Weg nach Jordanien migriert. Sein Sohn Uthman berichtet komplett abweichend davon, dass sein Vater zunächst zwei Jahre im Libanon gearbeitet habe, anschließend zwischen Syrien und dem Irak gependelt und von Syrien schließlich nach Jordanien migriert sei.

Die zurückbleibenden Familienmitglieder lebten im Haus von Abu Uthmans Vater. Durch die Abwesenheit des Familienvaters wurde die Position seiner Ehefrau und seiner Kinder gestärkt, was längerfristige Auswirkungen auf die Etablierung in Jordanien, aber auch auf Heiratsentscheidungen in der Familie haben sollte. Diese Aspekte werden im Interview aber weitgehend durch ein in diese Periode fallendes, tief einschneidendes Erlebnis überdeckt, über das die Familienmitglieder unabhängig voneinander detailliert berichten. Im Jahr 2009 heiratete im Familienanwesen, aus dem sie 2003 ausgezogen waren, Um Uthmans Schwester. In der angrenzenden schiitischen Nachbarschaft wurde an diesem Tag ein Selbstmordanschlag auf ein Café verübt, bei dem viele Menschen starben. Anschließend griffen schiitische Milizen ihre Nachbarschaft an. Sie drangen in das Anwesen ein und drohten den Frauen mit Vergewaltigung und mit der Ermordung der Männer. Uthman und ein Onkel flüchteten über die Felder, später flüchteten auch die Frauen. Im Interview ist die Zuwendung zu diesen Ereignissen brüchig. Die Töchter Samar und Aya gehen zwar auf unsere Angebote, die Ereignisse zu detaillieren, ein. Die Erzählungen sind aber von Pausen, Abbrüchen, der Verdichtung verschiedener Ereignisse geprägt und deuten traumatisches Erleben an. Samar erzählt von den vielen Leichen, die sie auf den Straßen habe liegen sehen, auch die eines Nachbarn. Aya berichtet, sie schlafe bis heute schlecht. Uthman beschreibt detailliert die Flucht durch Gräben und Felder, mit der ständigen Angst, entdeckt zu werden.

Es ist unklar, ob dieses Ereignis, der Tod von Abu Uthmans Vater kurz darauf oder eine von Bekannten angepriesene unproblematische Migrationsmöglichkeit in den Westen die kurz darauf beginnende schrittweise Migration der gesamten Kernfamilie nach Amman auslöste. Um Uthman zog mit ihren drei jüngsten Kindern 2010 zu Abu Uthman nach Amman. Der älteste Sohn Uthman hingegen pendelte 
noch einige Jahre zwischen Amman und Bagdad hin und her, bevor er 2014 endgültig nach Jordanien zog. Auch die älteste Tochter Samar kehrte noch 2010 nach Bagdad zurück, heiratete mit 14 Jahren einen schiitischen Cousin. Nachdem ihr Mann entführt oder ermordet worden war, migrierte Samar mit ihrer Tochter ca. 2012 nach Amman. Dort trat die Kernfamilie wie bei der Binnenmigration als Einheit zusammen und wurde zum Lebensmittelpunkt.

\section{Ankunft in Amman, Familienzusammenführung und Versuche der Etablierung}

Die Familienmitglieder zogen mit der Erwartung nach Amman, dass sie bald in die USA würden umsiedeln können. Sie sahen Amman wie viele irakische Geflüchtete als Zwischenstation. Diese Vorstellung speiste sich aus vorangegangenen Flüchtlingsbewegungen vom Irak nach Jordanien, als viele Geflüchtete zügig weitermigrieren konnten und diese Erfahrung über die größer werdenden Netzwerke an migrationswillige Iraker*innen zurücktrugen (Chatelard 2010a). Das als temporär erwartete Leben in Amman verstetigte sich jedoch im Falle der Familie Abu und Um Uthmans.

Dies lag einerseits an den rechtlichen Bedingungen, die die Immobilisierung in Jordanien fördern. Iraker*innen, die nach Ablauf ihres Visums illegalisiert in Jordanien bleiben, werden nicht deportiert, sondern als eine Art „Gäste“ angesehen (Mason 2011). Für jeden Tag fällt eine Gebühr von 1,5 jordanischen Dinaren an. Wer das Land verlassen möchte, kann nicht sicher sein, ob eine Wiedereinreise erlaubt ist, und ist zudem aufgefordert, die akkumulierten Gebühren zu begleichen. Als „Gäste“ erhalten die Geflüchteten (wenn überhaupt) nur Zugang zu grundlegenden Gesundheits- oder Bildungsangeboten. Sie dürfen offiziell nicht arbeiten, eine Aufenthaltserlaubnis kann nur mit einer hohen Bankeinlage erworben werden. Viele arbeiten prekär und unterbezahlt im informellen Sektor. Dieser legale Rahmen verhindert die Etablierung und katalysiert die Wahrnehmung von Ausgrenzung. Sie ermöglicht die Ausbeutung der Geflüchteten und befördert ihren sozialen Abstieg (Chatelard 2010a; El-Abed 2014; El Dardiry 2017; Mason 2011).

Andererseits war die Immobilisierung vor dem Hintergrund der legalen Bedingungen auch mit Erfahrungen verknüpft, die die Familienmitglieder bereits im Zuge der bisherigen transformatorischen Flucht gemacht hatten und die sie in Amman wieder erlebten: Auf die Erfahrung von struktureller Fremdheit und Mittellosigkeit reagierten die Eltern, indem sie die Kinder aus der Schule nahmen, um das Einkommen zu verbessern oder um sie zu verheiraten. Die Familienmitglieder hatten prekäre Jobs und wurden vom zuvor bäuerlich geprägten Leben und von der erweiterten Familie weiter distanziert. Die bestehenden, auch die familialen Verbindungen in den Irak verloren sich weiter. Man kann von einer Subproletarisierung der Familie sprechen. Im Zuge dessen verfestigten sich die bereits zuvor initiierten Machtverschiebungen innerhalb der Familie. Die Kernfamilie blieb als Fokus erhalten, und 
einzelne Familienmitglieder, die hätten weiter migrieren können, ließen sich nicht darauf ein. Die Familie konnte auch ihr soziales, kulturelles oder ökonomisches Kapital nicht erhöhen, um die Chancen auf Weiterreise zu steigern.

Géraldine Chatelard (2010b: 60) beschreibt, dass die Verarmung und das soziale Absinken der irakischen Geflüchteten im Verhältnis zur Menge an sozialen Verbindungen und zum Grad der Illegalität stehe, die die Geflüchteten haben. Chatelard hebt hervor, dass die Mitglieder dieser Gruppierung häufig durch familiale Unterstützung von außen, „überleben “ könnten. Doch die Familie Abu und Um Uthmans kann in Amman nicht einmal auf diese Unterstützung zurückgreifen. Die Familienmitglieder versuchen zwar zunehmend, die im Laufe der Flucht entstandene finanzielle Mittellosigkeit und die soziale Isolation zu überwinden. Aber wie wir ausführen werden, verfestigte sich die Position der Familie in der Ammaner Unterschicht gerade durch die Versuche, die prekäre Ankommensphase zu bewältigen.

Als die Familie in Amman eintraf, mietete sie eine Kellerwohnung in einem armen Viertel im Osten der Stadt. Bereits die Ankunft in Amman war für Abu Uthman mit ähnlichen Wahrnehmungen von Fremdheit wie in der irakischen Provinz Salah ad-Din verknüpft:

Als ich nach Amman kam, weißt du Neuankömmling, hatte ich gar keine Freunde, keine Verwandte, kein Land und sowas. Als ich ausstieg, als ich aus dem Auto auf dem Platz im Altstadtzentrum ausstieg, war ich verwirrt, wohin ich gehen soll. Ich war also verwirrt, wie ich leben werde.

Abu Uthman hatte nach seiner Ankunft zunächst als Tagelöhner gearbeitet, wurde aber häufig um seinen Lohn geprellt und litt unter den körperlichen Folgen früherer Verletzungen im Zuge von Flucht und Folter. Er war zeitweise nicht arbeitsfähig bzw. konnte keinen körperlich anstrengenden Tätigkeiten nachgehen. Seine Hilflosigkeit angesichts dieser Entwicklungen zeigte sich, wie Tochter Aya erzählt, in Ungeduld, Kontrollversuchen und Wutausbrüchen:

Er war sehr nett im Irak aber weil er in der Fremde ist und so, [die Fremde] änderte ihn. ... Bei Gott, mein Vater wird immer wütend, das Leben machte ihn so, dass er schnell wütend wird. [Das Leben] veranlasste, dass er so wurde. Aber normalerweise war mein Vater ganz im Gegenteil dazu lieb, und er hat uns sehr geliebt. Trotz allem liebt er uns noch, aber er steht unter Druck.

Aya, die sich angesichts ihres jungen Alters kaum an ihren Vater im Irak erinnern dürfte, kann diese „Verhaltensänderungen“ nicht auf dessen lange Flucht und die schwere Folter beziehen, obwohl sie nicht unwahrscheinliche Folgen davon sind.

Um Uthman war in einer Schneiderei beschäftigt. Sie arbeitete wohl zum ersten Mal außerhalb des Familienkontextes, was für sie eine neue Erfahrung war und wie es ihr selbst sehr wichtig war zu betonen - komplett ihrer Sozialisation im Herkunftsmilieu widersprach und bei ihr zunächst Hilflosigkeit auslöste. Zwei Kinder besuchten in Amman zunächst lokale Schulen, die Schule eines weiteren Sohnes verlangte die Vorlage einer Aufenthaltsgenehmigung, die die Familie nicht hatte. Die älteste in Amman anwesende Tochter Aya (2012 ca. zwölf Jahre alt) übernahm die 
Versorgung der jüngeren Geschwister, bekochte und tröstete sie, machte kleinere Ausflüge zum nahegelegenen Flughafen. Der Familie mangelte es in den ersten zwei Jahren in Amman auch an Dingen des täglichen Bedarfs. Die Kinder sprechen von durch Hunger geprägten Tagen. In einer lokalen Moschee erhielten sie Kleiderspenden. Die Familie war wie bereits in der Provinz Salah ad-Din weitgehend isoliert, auf sich gestellt und verblieb als Einheit. Obwohl es auch Beispiele von Solidarität gab, erlebten sie ihre Nachbarschaft als genauso fremd und ablehnend wie Salah ad-Din. Sie erzählen von abweisenden Nachbarn, die Müll vor der Souterrain-Wohnung deponiert und gefragt hätten, was sie als „Iraker“ hier wollten. Abu Uthman fasst die Verknüpfung von Diskriminierung, Fremdheitserfahrungen und legaler Situation folgendermaßen zusammen: „Abgesehen davon betrachte dich selbst im offenen Gefängnis. Weil du absolut keine Rechte hast. Wenn du mit einem sprichst, [sogar] mit einem Jungen auf der Straße, wird er dir sagen: Sei still, sonst lasse ich dich abschieben."

Seit 2011 bezog die Familie Geld von der UNHCR, wahrscheinlich aus dem Fonds zur Unterstützung der bedürftigsten Familien, ein Betrag, der aber nicht zum Leben ausreichte. ${ }^{7}$ Um ihre materielle Situation weiter zu verbessern, stiegen die Kinder verstärkt in die Versorgung ein. 2012 verließ die zwölfjährige Aya nach der 5. Klasse die Schule. Sie und die im selben Jahr aus dem Irak nach Amman hinzugestoßene Samar (16) begannen, in einer Schokoladenfabrik zu arbeiten. Ihre Beschäftigung war illegal und von Lohnprellung, Anfeindungen und Drohungen durch Kolleg*innen geprägt. Später arbeitete der jüngere Bruder Hassan in der gleichen Fabrik. Die Bildungskarrieren wurden der Sicherung des Grundeinkommens geopfert, und die Jobs waren sogar im lokalen Vergleich sehr prekär und schlecht bezahlt. Der älteste Sohn Uthman hatte die Schule nur noch sporadisch besucht. Bis 2014 pendelte er nach Bagdad und arbeitete sowohl dort als auch in Amman. Das Dilemma war folgendes: In Jordanien arbeitete er illegal, was mit der Gefahr der Entdeckung und Deportation, mit Lohnprellung und schlechter Behandlung verbunden war. Er arbeitete etwa nachts in einem fahrbaren Kaffeestand auf den Straßen Ammans. Im Irak arbeitete er legal und verdiente so viel, dass er die Familie in Amman unterstützen konnte. Er nutzte in Bagdad das familiale Netzwerk. Allerdings erlebte er auch, dass Familienmitglieder ihn ausgrenzten, und war Gefahren für Leib und Leben ausgesetzt. Aufgrund seines eindeutig sunnitischen Vornamens wurde er mehrfach bedroht.

Das Einkommen der Familie bildete sich dementsprechend aus dem der Kinder und der Mutter, zeitweise dem des Vaters sowie den Leistungen der UNHCR. Die Familie konnte es sich wegen der Anstrengungen leisten, innerhalb des als eher arm geltenden Ostammans in einen als etwas besser geltenden Stadtteil zu ziehen.

Der Kontakt zur Familie im Irak schwächte sich indessen weiter ab. In der prekären ersten Phase in Amman hatte Abu Uthman mehrfach seine Geschwister und

72018 wurden 32.500 Familien mit monatlichen Zahlungen unterstützt, der Betrag reichte von 80 bis 300 jordanischen Dinaren. Weniger als ein Zehntel waren irakische Familien, die Mehrzahl syrische Familien. Vgl. UNHCR 2019. 
die erweiterte Familie um finanzielle Unterstützung gebeten, was aber alle ablehnten. Die Familienmitglieder gingen davon aus, dass es eine Art Strafe sei, weil Abu Uthman Land und Haus aufgegeben habe, was seine Brüder, die alle ihr Land behalten hatten und über ausreichend ökonomisches Kapital verfügen, nicht akzeptiert hätten. Sie seien die einzigen Mitglieder der erweiterten Familie, die nicht in den Regionen Baquba oder Bagdad wohnten und daher von familialen Zusammenhängen isoliert. Wie stark die Entfremdung von der dortigen Familie ist, zeigt Um Uthmans Angabe, sie habe seit ihrer Ankunft nur einmal mit ihren Brüdern telefoniert. Die Situation der erweiterten Familie war zwar sozio-ökonomisch relativ stabil, aber weiterhin von der anhaltenden Gewaltordnung im Irak bestimmt. Mindestens drei Neffen von Abu und Um Uthman wurden bei Anschlägen oder im Kampf als Soldaten getötet. Abu Uthman galt weiterhin als gesucht. Die Tochter Samar, deren Ehemann verschwunden war, erhielt selbst in Amman Todesdrohungen, hinter denen sie die Entführer/Mörder ihres Ehemanns vermutet. Die sich abschwächenden Kontakte zur erweiterten Familie, die anhaltende Gewaltordnung und die Bedrohung Abu Uthmans und Samars sind Antworten auf die sich angesichts der prekären Situation in Amman stellende Frage, warum die Familie bzw. einige Familienmitglieder nicht (zumindest temporär) in den Irak zurückkehrten.

Der Zukunftshorizont der Familie blieb lange die Weiterreise in die USA. Der Antrag auf Umsiedelung, den die Familie beim UNHCR gestellt hatte, wurde aber zweimal wegen der Inhaftierung und Soldatenvergangenheit Abu Uthmans abgelehnt (das zweite Mal 2014). Familienmitglieder schlugen Angebote, ohne Abu Uthman zu migrieren, aus bzw. die anderen Familienmitglieder forderten die Ablehnung eines solchen Angebotes von ihnen ein. Es wurde zunehmend deutlich, dass sich die temporäre Situation in Amman verstetigen könnte.

Die sich langsam ändernde Orientierung manifestierte sich in zwei Heiraten von Kindern Abu und Um Uthmans in eine palästinensisch-jordanische Familie in Amman. Diese arme palästinensische Familie ist einer Art Subproletariat zuzurechnen, in der mehrere Mitglieder mit kriminellen Aktivitäten in Verbindung gebracht werden und andere massive Gewalterfahrungen gemacht haben. Sie ist auf den ersten Blick ,unter“ dem Status der Familie Abu und Um Uthmans. Damit verbunden ist die Sehnsucht, sich nach einer erschöpfenden Phase der Migration längerfristig zu verorten. Und sie schien der einzige Weg, um gewisse Ansprüche in der jordanischen Gesellschaft durchzusetzen. Uthman spekulierte darauf, dass er durch die Heirat ein sogenanntes jordanisches Familienbuch bekommen könnte und dadurch bessere Chancen auf eine Aufenthalts- oder Arbeitserlaubnis. Ausländische Männer von jordanischen Ehefrauen haben keinen Anspruch auf einen Aufenthaltsstatus (und schon gar nicht auf Staatsbürgerschaft).

Es gab keine andere Lösung, die mir nützlich ist oder mir eine Arbeitsstelle ermöglicht, außer dass ich heirate, um ein jordanisches Familienbuch zu bekommen ... Aber mein Vater stimmte nicht zu, dass ich eine Jordanierin heirate. Er wollte mir eine der Töchter meines Onkels väterlicherseits bringen. Aber meine Onkel im Irak halfen mir nicht. Sie standen mir nicht bei, keiner von ihnen nahm mich auf. Auf welcher Grundlage soll ich 
nun um die Hand einer ihrer Töchter bitten ... Außerdem will ich heiraten, um zu leben, und ich will nicht heiraten, um herumzuirren ... Da sagte ich [den zukünftigen Schwiegereltern]: Stimmt [der Heirat] zu, und ich werde das Land nicht verlassen ... Ich bin hier bei euch, ich heirate und bleibe hier in Amman.

Mit der Heirat geht erstmals eine klare Verortung Uthmans und auch der Familie in Amman einher. Zum Beispiel bestand die palästinensische Familie im Ehevertrag auf einer Klausel, die für eine Rückkehr Uthmans in den Irak eine hohe Geldstrafe vorsieht, was eine Remigration deutlich erschwert. Die Trennung von der Herkunftsregion und den familialen Netzwerken im Irak wird auch an der Erwähnung des Aushandlungsprozesses zwischen Uthman und seinem Vater deutlich. Verschiedene Familienmitglieder erzählen, dass sich Abu Uthman mit seiner Absicht, Uthman dem familialen Handlungsmuster folgend mit einer Cousine väterlicherseits zu verheiraten und damit einem gängigen Heiratsmuster in der Familie zu entsprechen, nicht gegenüber seinem Sohn und seiner Frau durchsetzen konnte. Mit Verweis auf die ausbleibende Hilfe durch die Familie und den schwindenden Kontakt überzeugten Uthman und seine Mutter Abu Uthman von der Heirat in Amman. Das wiederum vergrößerte die Distanz zu irakischen Familienteilen. Die Heiraten verdeutlichen den Wandel in der innerfamilialen Machtbalance: Wir hatten bereits angesprochen, dass Abu Uthman körperlich beeinträchtigt war, die Kinder bei der Familienversorgung halfen, die Ehefrau zum Familieneinkommen beitrug und im öffentlichen Raum auftrat. Die Kinder ordneten sich nicht mehr unhinterfragt der väterlichen Autorität unter. Es ist unwahrscheinlich, dass dies in Diyala in ähnlicher Form möglich gewesen wäre.

Diese Konstellation wiederholte sich bei der zweitältesten Tochter Aya, die zwei Jahre später einen Bruder von Uthmans Frau heiratete. Mit diesen Heiraten stellte sich die Familie auf Amman als längerfristigen Lebensmittelpunkt ein. Einerseits wurden die prekären Lebensverhältnisse durch den Charakter der eingeheirateten Familie festgeschrieben: Sie ist bereits in dem Milieu angesiedelt, in das die Familie Abu und Um Uthmans absinkt. Andererseits ist die Hochzeit eine der wenigen Möglichkeiten, die eigenen Lebenschancen zu verbessern, da weder Naturalisierung noch Bildungsaufstieg, Weitermigration, Netzwerk oder Rückkehr als Optionen zur Verfügung stehen.

\section{Zusammenfassung}

In unserem Beitrag haben wir aufgezeigt, inwiefern die Verankerung in einem lokalisierten Milieu vor Beginn der (Flucht-)Migration in eine stark transformatorische Fluchtdynamik münden kann. Wir haben einen typischen Zusammenhang von lokalisierten Geflüchteten und transformatorischen Fluchtprozessen skizziert, den wir exemplarisch am Fall der Familie Abu und Um Uthmans aufgezeigt haben. Die zentrale Dynamik und deren wesentliche Komponenten seien an dieser Stelle kurz zusammengefasst: Die Familie verließ im Verlauf der Flucht - in Etappen und mit 
Pendelbewegungen - ihren zuvor auf das nahe lokale und regionale Umfeld begrenzten Mobilitätsraum, der ihre „Welt in potentieller Reichweite“ ausmachte (Schütz/Luckmann 2003[1979/1984]: 71 ff.). Diese war unter anderem charakterisiert durch einen am bäuerlichen Milieu orientierten Handlungshorizont sowie durch die Bindung an die erweiterte Familie und an das weitere verwandtschaftliche Netzwerk. Die Fluchtdynamik und das Fluchterleben waren dadurch strukturiert, dass sich die Familienmitglieder der Gewalt nicht innerhalb ihrer Welt in potentieller Reichweite bzw. ihres „gewohnten“ Mobilitätsraums entziehen konnten. Aber mit der (wiederholten) Flucht über diesen Raum hinaus war massives Fremdheitserleben verknüpft. Das Gewohnheitswissen funktionierte bereits in einer angrenzenden Provinz innerhalb der staatlichen Grenzen nicht mehr, Handlungspläne wurden unsicher, weil die Familienmitglieder nicht zum „üblichen“ (stark bäuerlich geprägten) Modus zurückkehren konnten und sich von den lokalen Einwohner*innen ausgeschlossen fühlten. Schließlich ließen im Verlauf der Flucht die Bindungen an die familiale Großgruppe und die Verankerung im Herkunftsmilieu nach. Die Migration außerhalb des lokalen Mobilitätsraumes führte nicht - wie man hätte erwarten können - zu einer Erweiterung des familialen oder sozialen Netzwerks (im Sinne einer Pionierleistung), sondern eher zur Ablösung von erweiterter Familie und weiterem verwandtschaftlichen Netzwerk. Gleichzeitig wurde der Verbund der Kernfamilie zum primären Orientierungspunkt der Familienmitglieder. Diese Dynamik prekarisierte die Situation der Familie sowohl innerhalb des Iraks als auch in Jordanien, wo die Familie ohnehin rechtlich-administrativ marginalisiert war. Sie war jedoch zudem verknüpft mit einer Veränderung der familialen Machtbalance zugunsten von Ehefrau und Kindern und zuungunsten Abu Uthmans, der durch Gewalt und Flucht körperlich und seelisch besonders versehrt war.

Generell verdeutlicht der rekonstruierte Typus, inwiefern Flucht Transformationen, Anpassungsleistungen und handlungspraktische Herausforderungen einfordert, wenn sie mit dem (sukzessiven) Verlust eines Milieus einhergeht, das unter anderem durch nahräumliche Verortung und die intergenerationell weitergegebene Bedeutung des Bodens verbunden ist. Im Gegensatz dazu ist Flucht im Kontext eines Milieus, in dem transnationale bzw. translokale Verbindungen selbstverständlich sind, weniger von diesen Herausforderungen geprägt (vgl. Becker im Druck). Die Relevanz lokaler Verortung für Fluchtdynamik und -erleben wurde im Falle der Familie Abu und Um Uthmans bereits bei der Migration in eine angrenzende Provinz innerhalb des Nationalstaates deutlich.

Zuletzt möchten wir den von uns rekonstruierten Typus auf das in der Einleitung diskutierte „regionalist paradigm“ beziehen. Es beschreibt Flucht- und Migrationsprozesse im Nahen Osten als besonders strukturiert durch historisch etablierte translokale und transnationale Verbindungen und soziale Netzwerke, die sich über verschiedene Kontexte ausdehnen. Doch in der exemplarisch diskutierten Fallrekonstruktion wurde deutlich, dass die Flucht in geographisch angrenzende und kulturell ähnlich erscheinende regionale Zusammenhänge das Erleben eines Zusammenbruchs etablierter lebensweltlicher Strukturen, Kapitalentwertung und einen 
erheblichen sozialen Abstieg nicht notwendigerweise ausschließt. Unsere Analyse ergänzt daher das „,regionalist paradigm“. Auf Basis unserer empirischen Analysen haben wir einen Typus erörtert, der sich durch vormals stark lokalisierte Verortung und einen anschließenden stark transformatorischen Fluchtprozess auszeichnet. Er verdeutlicht, inwiefern Ausmaß von translokaler und transnationaler Mobilität bereits vor der Flucht, (sich wandelnde) familiale und soziale Netzwerke im Fluchtoder Ankunftskontext und die Struktur des Fluchtprozesses (direkt oder indirekt, komplex und in seiner Gesamtheit ungeplant) in Zusammenhang stehen. Diese Differenzierung hilft uns dabei, die unterschiedlichen Erfahrungsräume und gesellschaftlichen Positionen von Geflüchteten und Migrant*innen in Amman zu verstehen und zu erklären. Diese drücken sich nicht nur in ihren rechtlich-administrativen Ankommensbedingungen oder in ihrer relativen Stellung zueinander aus, sondern unterscheiden sich auch in ihrer translokalen oder lokalen Verortung vor der Flucht und im Charakter des Fluchtprozesses. Über Amman und den Nahen Osten hinausgehend erwarten wir, dass die Rekonstruktion des Zusammenhangs von lokalisierter Verortung und transformatorischen Fluchtprozessen wie des Verhältnisses von translokalen und stark lokalisierten Familien einen Beitrag zum Verständnis der Erfahrungsräume und gesellschaftlichen Positionen von Geflüchteten leisten kann.

\section{Literatur}

Becker, J. (im Druck): Refugees, Migration and the Tightening Borders in the Middle East. Perspective from Biographical Research on the Re-Figuration of Spaces and Cross-Cultural Comparison. In: Baur, N./Mennell, S./Million, A. (Hrsg.): The Re-Figuration of Spaces and Cross-Cultural Comparison. Forum: Qualitative Social Research.

Becker, J./Hinrichsen, H./Worm, A. (im Druck): Fluchtmigration von Syrien nach Jordanien im familiengeschichtlichen und regionalhistorischen Kontext. Zum Nutzen einer biographietheoretischen und figurationssoziologischen Forschungsperspektive. In: Köttig, M./Witte, N. (Hrsg.): Biographie und Kollektivgeschichte. Weinheim: Beltz Juventa.

BenEzer, G./Zetter, R. (2015): Searching for Directions. Conceptual and Methodological Challenges in Researching Refugee Journeys. In: Journal of Refugee Studies, 28(3), 297-318.

Chatelard, G. (2010a): What Visibility Conceals. Re-Embedding Refugee Migration from Iraq. In: Chatty, D./Finlayson, B. (Hrsg.): Dispossession and Displacement. Forced Migration in the Middle East and Africa. Oxford: Oxford University Press, 17-44.

Chatelard, G. (2010b): Cross-Border Mobility of Iraqi Refugees. In: Forced Migration Review, 34, 60-61. 
El-Abed, O. (2014): The Discourse of Guesthood. Forced Migrants in Jordan. In: Häusermann Fábos, A./Isotalo, R. (Hrsg.): Managing Muslim Mobilities. New York: Palgrave Macmillan, 81-100.

El Dardiry, G. (2017): "People Eat People”. The Influence of Socioeconomic Conditions on Experiences of Displacement in Jordan. In: International Journal of Middle East Studies, 49(4), 701-719.

Elwert, G./Evers, H.-D./Wilkens, W. (1983): Die Suche nach Sicherheit.

Kombinierte Produktionsformen im sogenannten informellen Sektor. In: Zeitschrift für Soziologie, 12(4), 281-296.

Etzold, B. (2019): Auf der Flucht. (Im)Mobilisierung und (Im)Mobilität von Schutzsuchenden. State-of-Research Papier 04, Verbundprojekt ,Flucht: Forschung und Transfer'. Institut für Migrationsforschung und interkulturelle Studien (IMIS) der Universität Osnabrück/Bonn: Internationales Konversionszentrum Bonn (BICC).

Freitag, U./von Oppen, A. (2010): Introduction. Translocality. An Approach to Connection and Transfer in Regional Studies. In: Freitag, U./von Oppen, A. (Hrsg.): Translocality. The Study of Globalising Processes From a Southern Perspective. Leiden/Boston: Brill, 1-22.

Haddad, F. (2013): Sectarian Relations in Arab Iraq. Contextualising the Civil War of 2006-2007. In: British Journal of Middle Eastern Studies, 40(2), 115-138.

Mason, V. (2011): The Im/Mobilities of Iraqi Refugees in Jordan. Pan-Arabism, 'Hospitality' and the Figure of the 'Refugee'. In: Mobilities, 6(3), Special issue: Mobilities and Forced Migration, 353-373.

Neubert, D./Stoll, F. (2015): Socio-Cultural Diversity of the African Middle Class. The Case of Urban Kenya. Bayreuth African Studies Working Papers 14: Institute of African Studies (IAS) at the University of Bayreuth. Online: https:// epub.uni-bayreuth.de/2500/ <26.02.2020>.

Rehbein, B./Stoll, F. (2017): Mittelschichten und Ungleichheit im Globalen Süden. In: Burchardt, H.-J./Peters, S./Weinmann, N. (Hrsg.): Entwicklungstheorie von heute. Entwicklungspolitik von morgen. Baden-Baden: Nomos, 111-132.

Rosenthal, G./Bahl, E./Worm, A. (2016): Illegalisierte Migrationsverläufe aus biografietheoretischer und figurationssoziologischer Perspektive. Die Landgrenze zwischen Spanien und Marokko. In: Forum Qualitative Sozialforschung, 17(3), Art. 10. Online: www.qualitativeresearch.net/index.php/fqs/article/view/2686/4012<05.05.2020>.

Schütz, A./Luckmann, T. (2003[1979/1984]): Strukturen der Lebenswelt. Konstanz: UVK. 
Shami, S. (1996): Transnationalism and Refugee Studies. Rethinking Forced Migration and Identity in the Middle East. In: Journal of Refugee Studies, 9(1), $3-26$.

The Oklahoman (2007): Residents. Baqouba is Deadly Ghost City, 22. Januar 2007. Online: https://oklahoman.com/article/3002595/residents-baqouba-isdeadly-ghost-city $<26.02 .2020>$.

UNHCR (2019): Multi-Purpose Cash Assistance. 2018 Post Distribution Monitoring Report. Online: https://data2.unhcr.org/en/documents/download/68296<26.02.2020>.

Waldenfels, B. (1997): Topographie des Fremden. Frankfurt a. M.: Suhrkamp.

Worm, A. (2019): Fluchtmigration aus Syrien. Eine biographietheoretische und figurationssoziologische Studie. Göttinger Beiträge zur soziologischen Biographieforschung, Bd. 1. Göttingen: Universitätsverlag Göttingen. Online: https://doi.org/10.17875/gup2019-1228<09.04.2020>. 